\title{
Effect of short-term inhalation of warm saline atomised gas on patients with non-cystic fibrosis bronchiectasis
}

\author{
Lihong Zhong ${ }^{1,4}$, Ying Xiong ${ }^{2,4}$, Zeguang Zheng ${ }^{3,4}$, $\mathrm{Ni} \mathrm{Liu}^{3}$, Jieying $\mathrm{Hu}^{3}$, \\ Feng Yang ${ }^{3}$ and Rongchang Chen $^{3}$
}

Affiliations: 'Luoding People's Hospital, Yunfu, China. ${ }^{2}$ Wuhan Fourth Hospital, Puai Hospital, Tongji Medical College, Huazhong University of Science and Technology, Wuhan, China. ${ }^{3}$ State Key Laboratory of Respiratory Diseases, National Clinical Research Center for Respiratory Disease, Guangzhou Institute of Respiratory Health, the First Affiliated Hospital of Guangzhou Medical University, Guangzhou, China. ${ }^{4}$ These authors contributed equally.

Correspondence: Zeguang Zheng, State Key Laboratory of Respiratory Diseases, National Clinical Research Center for Respiratory Disease, Guangzhou Institute of Respiratory Health, the First Affiliated Hospital of Guangzhou Medical University, 151 Yanjiang Rd, Guangzhou, China. E-mail: 135030340121a139.com

\section{ABSTRACT}

Objective: Secretion clearance is advocated in non-cystic fibrosis bronchiectasis, but is often neglected in clinical treatment. The present study aimed to investigate the effect of inhaled $0.9 \%$ normal saline by ultrasonic nebuliser with warming (UNW) in stable non-cystic fibrosis bronchiectasis patients with purulent sputum.

Methods: 27 stable patients were enrolled in a randomised crossover trial comprising 3 months of daily UNW aerosol therapy compared with 3 months of daily oral expectorant treatment. The outcomes were quality of life (assessed via the Leicester cough questionnaire (LCQ)), sputum characteristics, pulmonary function, 6-min walk distance (6MWD) and acute exacerbation frequency.

Results: Compared with baseline, the sputum viscosity, sputum colour, LCQ score and 6MWD were significantly improved with UNW ( $\mathrm{p}<0.05)$, and the improvements in sputum colour, LCQ score, 6MWD and inspirational capacity with UNW were significantly better than those achieved via treatment with oral expectorant $(\mathrm{p}<0.05)$. There were no significant differences between treatments in pulmonary function, sputum cytology classification and inflammatory factors of sputum.

Conclusion: UNW aerosol therapy in non-cystic fibrosis bronchiectasis has small yet significant benefits. However, UNW does not improve the degree of inflammation.

@ERSpublications

Curative effect of ultrasonic nebulisation with warming for stable non-cystic fibrosis bronchiectasis patients with sputum: a randomised crossover study http://bit.ly/2RjyfET

Cite this article as: Zhong L, Xiong Y, Zheng Z, et al. Effect of short-term inhalation of warm saline atomised gas on patients with non-cystic fibrosis bronchiectasis. ERJ Open Res 2020; 6: 00130-2019 [https://doi.org/10.1183/23120541.00130-2019].

This study is registered at www.chictr.org.cn with identifier number ChiCTR-IPR-15006911. If other researchers need the research data, they can contact the author to obtain it by e-mail.

Received: 24 May 2019 | Accepted after revision: 27 Nov 2019

Copyright $\odot$ ERS 2020. This article is open access and distributed under the terms of the Creative Commons Attribution Non-Commercial Licence 4.0. 


\section{Introduction}

Non-cystic fibrosis bronchiectasis (NCFB) is a chronic lung disease characterised by permanent dilation of the bronchi. Recurrent chronic airway inflammation caused by suppurative infection leads to continuous lung tissue damage and pulmonary dysfunction characterised by chronic coughing and abnormal sputum production, sometimes accompanied by haemoptysis, and may lead to respiratory failure and chronic cor pulmonale [1]. The predominant feature of bronchiectasis is purulent sputum. The drainage and clearance of purulent sputum are closely related to the progression and recurrent acute exacerbation of the disease [2].

The timely clearance of airway secretions is important when treating bronchiectasis. For patients with a spontaneous cough, the ability to clear airway secretions is mainly affected by cilia movement and sputum viscosity [3]. The motility of the cilia is related to the temperature and humidity of the airways. The fluidity of sputum can be improved via various methods, such as the administration of expectorant drugs [4], adequate hydration, instillation of normal saline into the airway [5] and inhalation of supersaturated gas [6]. Dilute sputum is then pushed from the peripheral airways to the central airway by the directional movement of the cilia on the surface of the respiratory mucosa, and the secretions are removed by coughing. In patients with acute exacerbation of chronic obstructive pulmonary disease, ultrasonic nebulisation with warming (UNW) and oxygenation promotes expectoration of sputum with fewer adverse reactions and a higher level of comfort than ultrasonic nebulisation and oxygen-driven nebulisation alone. This is because ultrasonically atomised gas at a condition of $37^{\circ} \mathrm{C}$ and $100 \%$ relative humidity not only fully dilutes the sputum, but also provides the optimal environment for mucosal cilia [7]. However, no study has evaluated the application of this technology to bronchiectasis patients with structural lung damage. The present study aimed to investigate whether inhaled $0.9 \%$ normal saline by ultrasonic nebuliser had an effect in stable NCFB patients with purulent sputum.

\section{Methods}

Patients

Patients with NCFB were recruited from the First Affiliated Hospital of Guangzhou Medical University (Guangzhou, China) between December 2014 and December 2016. Patients were diagnosed with bronchiectasis based on clinical features and high-resolution computed tomography [8]. Participants were eligible for inclusion if they were aged $>18$ years, clinically stable, daily produced $>10 \mathrm{ml}$ of purulent sputum and had no smoking history or had quit smoking for $>1$ year. The exclusion criteria were 1) study participation considered likely by a researcher to put the patient at risk; 2) concomitant serious primary diseases of the heart, liver, kidney, haematopoietic system and/or other important organs or systems; and 3) cognitive impairment, or other conditions that may prevent the patient from cooperating with the investigators. Written informed consent was obtained before inclusion. The study was approved by the ethics committee of the First Affiliated Hospital, Guangzhou Medical University, and is registered in the Chinese Clinical Trial Registry (www.chictr.org.cn, identifier number ChiCTR-IPR-15006911).

\section{Study design}

This was a randomised crossover trial of 3 months of daily UNW therapy followed by a 2-week washout period, compared with 3 months of daily oral expectorant treatment in patients with NCFB. UNW aerosol therapy was performed by inhaling $0.9 \%$ normal saline for 20 min using an ultrasonic nebuliser atomiser (vgr001 heating ultrasonic atomiser, Guangzhou Institute of Respiratory Health, First Affiliated Hospital of Guangzhou Medical University and Dongguan Yongsheng Medical Products, Guangzhou, China; registration certificate number 20172231068). The equipment provides ultrasound atomised gases with additional warming and oxygen. Its aerosol output is $2 \mathrm{~mL} \cdot \mathrm{min}^{-1}$; the diameter of its aerosolised particles is $\sim 1-5 \mu \mathrm{m}$, its temperature is $\sim 32-35^{\circ} \mathrm{C}$ at the outlet and oxygen flow rate can be adjusted from 0 to $10 \mathrm{~L} \cdot \mathrm{min}^{-1}$. The patients received 20 -min UNW treatment in a sitting position three times a day. Oral expectorant therapy constituted $30 \mathrm{mg}$ ambroxol hydrochloride three times a day. Both treatments were provided as add-on to existing NCFB therapy.

\section{Compliance}

Patients were followed-up by telephone twice a week and were asked to report the treatments they had performed and their symptoms.

\section{Measurement}

The study design and assessment time points are shown in figure 1.

\section{Leicester cough questionnaire}

Health-related quality of life (HRQoL) was assessed using the Leicester cough questionnaire (LCQ) [9]. The LCQ has three domains: physical, psychological and social (each domain score ranges from 1 to 7 , 


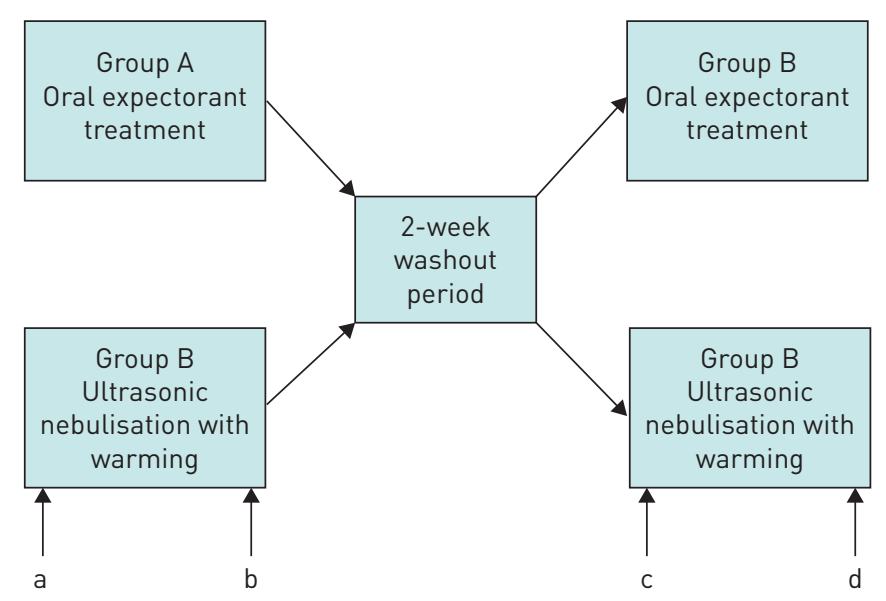

FIGURE 1 Study protocol. Assessments were performed at the start and end of each treatment period. Assessment time-points: a) before study; bl after 3 months of treatment; cl after washout period; d) after 3 months of alternative treatment.

with higher scores indicating better HRQoL). The minimal important differences for the LCQ are 0.2 for the physical domain, 0.2 for the social domain, 0.8 for the psychological domain and 1.3 for the total score.

\section{Sputum characteristics}

The assessed sputum characteristics included the 24 -h sputum volume, purulent score and viscosity. The 24-h sputum volume was determined by the average of all 3 days' sputum before each follow-up. Fresh sputum was obtained from each patient after they had cleaned their mouth and coughed effectively. Sputum purulence was scored by the same experienced technician as 0 (no sputum), 1 (completely transparent), 2 (almost transparent), 3 (translucent but colourless), 4 (opaque and milky white), 5 (grey), 6 (pale green), 7 (moderately green) or 8 (dark green) [10]. Sputum viscosity was evaluated by fluidity of sputum on vessel wall as 1 (sputum adherent to the vessel wall and does not slide), 2 (slides slowly under the action of gravity), 3 (falls under gravity) or 4 (slides easily with a small amount of mucus attached)

\section{Pulmonary function}

Spirometry was performed using a Pony FX pulmonary function instrument (COSMED, Rome, Italy). The forced expiratory volume in $1 \mathrm{~s}\left(\mathrm{FEV}_{1}\right)$, forced vital capacity $(\mathrm{FVC}), \mathrm{FEV}_{1} \%$ predicted, $\mathrm{FEV}_{1} / \mathrm{FVC}$ ratio, inspiratory capacity and peak expiratory flow were assessed at rest.

\section{Exercise endurance}

Exercise endurance was assessed using 6-min walk distance (6MWD) [11].

\section{Disease exacerbation}

An exacerbation was defined as persistent $(>24 \mathrm{~h})$ deterioration of at least three respiratory symptoms (including cough, dyspnoea, haemoptysis, increased sputum purulence or volume, and chest pain), with or without fever $\left(>37.5^{\circ} \mathrm{C}\right)$, radiographic deterioration, systemic disturbances or deterioration in chest signs. Patients reported the changes voluntarily when their respiratory symptoms deteriorated, and were then assessed by the clinicians to confirm the presence of an exacerbation.

\section{Cell classification of sputum}

The sputum was incubated at $37^{\circ} \mathrm{C}$ with $0.1 \%$ dithiothreitol solution for $30 \mathrm{~min}$. The supernatant was discarded after centrifugation, and the remaining particles were then mixed with the same amount of normal saline. The cells were stained, counted and classified using light microscopy. The percentages of eosinophils, neutrophils, lymphocytes and macrophages were calculated.

\section{Detection of inflammatory factors in sputum supernatant}

The concentrations of interleukin (IL)-6, IL-8 and tumour necrosis factor (TNF)- $\alpha$ in the sputum supernatant were determined using ELISA conducted in strict accordance with the manufacturer's instructions (Human TNF- $\alpha$ Quantikine HS ELISA Kit HSTA00D, Human IL-8/CXCL8 Quantikine HS ELISA Kit HS800, Human IL-6 Quantikine HS ELISA Kit HS600B, R\&D Systems, Minneapolis, MN, USA). 
Safety

Patients were monitored for exacerbation of symptoms and all other medical events throughout the study period.

\section{Statistical methods}

Statistical analyses were performed using the Statistical Package for the Social Sciences (version 19.0; SPSS, Chicago, IL, USA). The two-sample t-test was used to assess the statistical significance of changes between the study groups at baseline and the assessment time points. ANOVA with repeated measurements was applied to analyse the changes in continuous parameters over time between treatment periods. All tests were two-tailed, and $\mathrm{p}$-values of $\leqslant 5 \%$ were considered statistically significant.

\section{Results}

\section{Study population}

The final analysis included data from 27 patients who completed both treatment cycles (figure 2). Table 1 summarises the baseline characteristics of these patients.

\section{Treatment differences}

UNW resulted in significant improvements in the social, psychological and total scores of the LCQ compared with baseline (table 2, figure 3). UNW resulted in a significantly better purulent score and inspiratory capacity compared with oral expectorant treatment (table 2). In addition, UNW achieved significantly better exercise capacity than oral expectorant treatment (figure 4). The 6MWD after UNW aerosol therapy was significantly longer than that before treatment $(503.63 \pm 105.67 \mathrm{~m}$ versus $529.48 \pm 115.67 \mathrm{~m}, \mathrm{p}<0.01$ ), and the improvement value was significantly greater than that following oral expectorant therapy $(25.54 \pm 38.04 \mathrm{~m}$ versus $-24.30 \pm 78.73 \mathrm{~m}, \mathrm{p}<0.01)$. However, there were no significant differences between treatments in sputum cytological classification, $\mathrm{FEV}_{1}, \mathrm{FVC}, \mathrm{FEV}_{1} \%$ pred, $\mathrm{FEV}_{1} / \mathrm{FVC}$ ratio, peak expiratory flow or inflammatory factors in sputum supernatant (table 2).

\section{Exacerbation}

The frequency of acute exacerbation events in 3 months among patients who received UNW treatment and oral expectorant treatment were $0.41 \pm 0.69$ and $0.67 \pm 0.73$, respectively $(Z-1.414, p>0.05)$; it was lower than at baseline $(0.85 \pm 0.91)$ in patients who received UNW treatment $(Z-2.398, p<0.05)$.

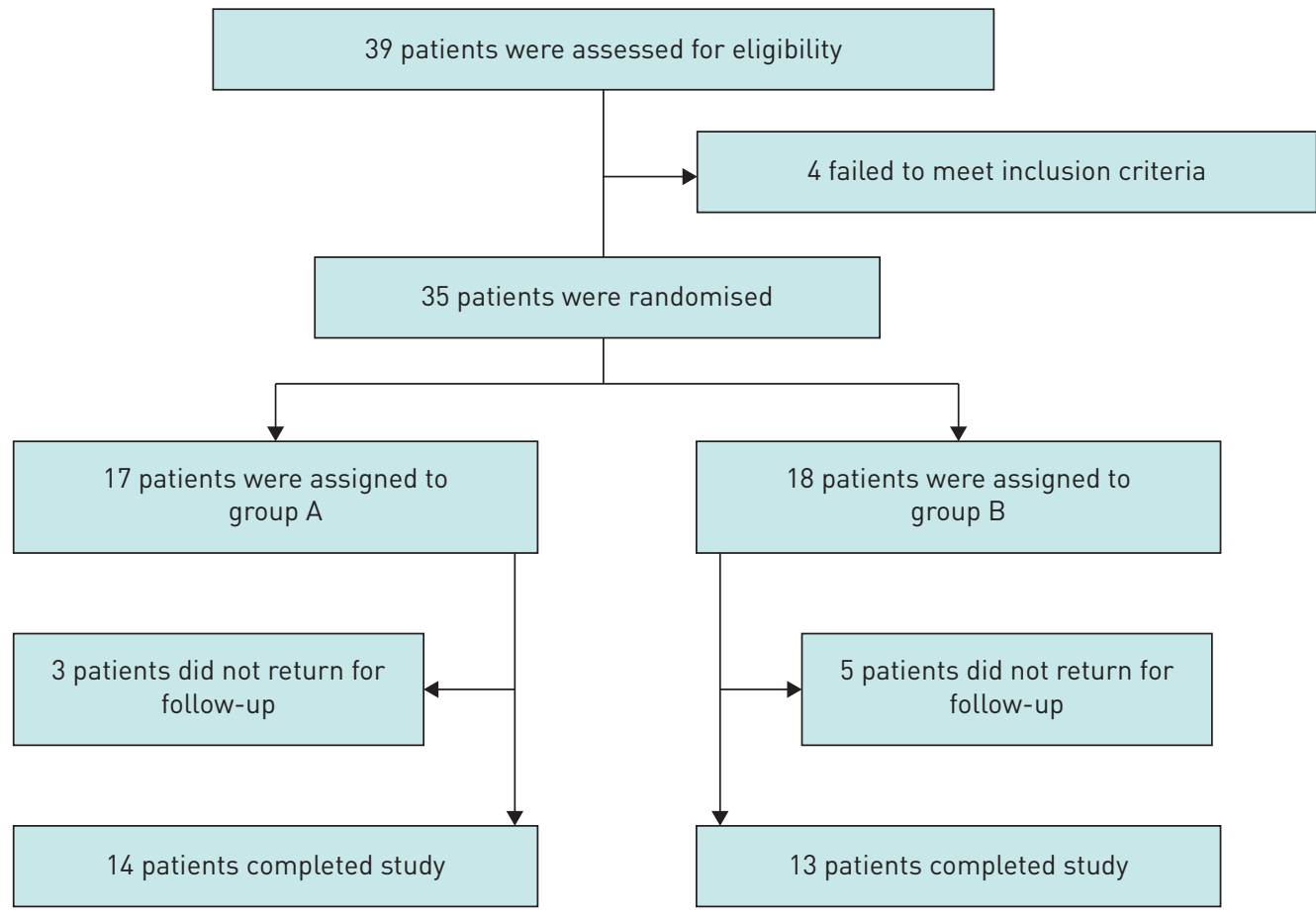

FIGURE 2 Screening, randomisation and follow-up. 


\section{TABLE 1 Baseline characteristics}

\begin{tabular}{|c|c|c|c|c|c|}
\hline & Group A & Group B & t-value & Z-value & p-value \\
\hline Subjects $\mathrm{n}$ & 14 & 13 & & & \\
\hline Age years & $51.64 \pm 19.93$ & $52.64 \pm 16.94$ & -0.28 & & 0.978 \\
\hline Sputum volume $\mathrm{mL} \cdot 24 \mathrm{~h}^{-1}$ & $57.64 \pm 56.91$ & $43.50 \pm 28.76$ & 0.741 & & 0.465 \\
\hline Purulent score & $6.14 \pm 1.16$ & $6.23 \pm 0.73$ & -0.233 & & 0.818 \\
\hline Sputum viscosity & $2.14 \pm 0.77$ & $2.36 \pm 1.00$ & -0.473 & & 0.640 \\
\hline FVC L & $2.47 \pm 1.07$ & $2.21 \pm 0.91$ & 0.685 & & 0.50 \\
\hline FVC $\%$ predicted & $66.43 \pm 13.78$ & $68.94 \pm 21.68$ & -0.419 & & 0.679 \\
\hline $\mathrm{FEV}_{1} /$ FVC $\%$ & $68.29 \pm 13.68$ & $74.50 \pm 10.32$ & -1.590 & & 0.125 \\
\hline PEF L & $4.38 \pm 2.05$ & $3.73 \pm 1.01$ & 0.925 & & 0.364 \\
\hline Inspiratory capacity L & $1.73 \pm 0.82$ & $1.47 \pm 0.56$ & 0.851 & & 0.403 \\
\hline 6MWD m & $507.64 \pm 130.51$ & $508.50 \pm 73.61$ & 0.122 & & 0.904 \\
\hline Total score & $12.12 \pm 2.95$ & $10.15 \pm 2.87$ & 1.779 & & 0.087 \\
\hline Bacteria count of sputum $\times 10^{4} \cdot \mathrm{mL}^{-1}$ & $795.83 \pm 395.25(n=12)$ & $836.42 \pm 550.75(n=11)$ & -0.380 & & 0.708 \\
\hline \multicolumn{6}{|l|}{ Sputum cell classification } \\
\hline Neutrophils \% & $94.83 \pm 8.09$ & $96.28 \pm 3.58$ & & -0.412 & 0.680 \\
\hline Macrophages \% & $2.96 \pm 7.41$ & $1.56 \pm 2.96$ & & -0.908 & 0.364 \\
\hline Eosinophils \% & $0.83 \pm 1.14$ & $0.78 \pm 1.17$ & & -0.461 & 0.645 \\
\hline Lymphocytes \% & $1.54 \pm 1.59$ & $1.39 \pm 2.22$ & & -0.129 & 0.897 \\
\hline TNF $-\alpha \times 10^{3} \mathrm{pg} \cdot \mathrm{mL}^{-1}$ & $1.91 \pm 2.25(n=12)$ & $1.00 \pm 1.36(n=5)$ & & -0.949 & 0.343 \\
\hline $\mathrm{IL}-8 \times 10^{3} \mathrm{pg} \cdot \mathrm{mL}^{-1}$ & $420.33 \pm 389.21(n=11)$ & $184.56 \pm 107.42(n=5)$ & & -0.108 & 0.914 \\
\hline $\mathrm{IL}-6 \times 10^{3} \mathrm{pg} \cdot \mathrm{mL}^{-1}$ & $0.52 \pm 0.82(n=7)$ & $1.46 \pm 1.03(n=5)$ & & -1.218 & 0.223 \\
\hline
\end{tabular}

Data are presented as $n$ or mean $\pm S D$, unless otherwise stated. $F E V_{1}$ : forced expiratory volume in $1 \mathrm{~s}$; FVC: forced vital capacity; PEF: peak expiratory flow; 6MWD: 6-min walk distance; LCQ: Leicester cough questionnaire; TNF: tumour necrosis factor; IL: interleukin.

\section{Safety}

Gastrointestinal reactions occurred in two patients after oral expectorant treatment, and disappeared after withdrawal; chest tightness occurred in one patient during UNW treatment, and disappeared after inhalation of short-acting bronchodilator.

\section{Discussion}

The HRQoL of patients with bronchiectasis is seriously affected by coughing and sputum production due to repeated infection. The so-called "vicious cycle hypothesis" was first proposed in 1986 [12]. Bacteria colonise in the sterile lower respiratory tract, causing chronic inflammation of the respiratory epithelium. Inflammatory mediators and hydrolases subsequently damage the alveolar tissue, destroy the elastic fibrous cartilage in the bronchial wall and impair the supporting function of the bronchial wall. Furthermore, the chronic inflammation impairs the function of the mucosal cilia epithelium of the airways. The bronchial wall then gradually expands due to coughing. The function of airway clearance is reduced or even lost, leading to phlegm retention in the dilated bronchi. As a result, chronic airway inflammation and repeated respiratory infections reduce local lung defence and form a vicious cycle of infection-inflammation-injury of the airway. In order to break this cycle, it is important to clear the remaining sputum in the respiratory tract [13]. Due to the thickening of airway wall in patients with bronchiectasis, it is difficult for drugs to reach the airway mucosa through the thickened airway wall and enter the airway to decompose sputum.

Factors affecting clearance of airway secretions include airway mucosal ciliary motility, sputum viscosity and airway patency. When exposed to saturated humidified gases close to body temperature (i.e. temperature $37^{\circ} \mathrm{C}$, relative humidity $100 \%$, absolute humidity $44 \mathrm{mg} \cdot \mathrm{L}^{-1}$ ), airway mucosal cilia swing best $[14,15]$. The simplest and most effective way to improve the fluidity of airway secretion is to directly increase the humidity of the gas entering the airway through atomisation inhalation. When the absolute humidity of the gas exceeds the humidity of the sputum surface, the sputum absorbs the moisture from the gas and becomes less viscous. 
TABLE 2 Therapeutic effect and comparison

\begin{tabular}{|c|c|c|c|c|c|c|c|c|c|c|c|c|}
\hline & \multicolumn{4}{|c|}{ UNW } & \multicolumn{4}{|c|}{ Oral expectorant } & \multicolumn{4}{|c|}{$\begin{array}{l}\text { Comparison of the difference between the two } \\
\text { treatments }\end{array}$} \\
\hline & Pre-therapy & $\begin{array}{l}\text { After } 3 \text { months } \\
\text { of treatment }\end{array}$ & $\mathrm{t}$-value & p-value & Pre-therapy & $\begin{array}{l}\text { After } 3 \text { months } \\
\text { of treatment }\end{array}$ & t-value & p-value & UNW & $\begin{array}{c}\text { Oral } \\
\text { expectorant }\end{array}$ & F-value & p-value \\
\hline FVC L & $2.29 \pm 0.92$ & $2.34 \pm 0.97$ & -0.756 & 0.457 & $2.36 \pm 0.98$ & $2.34 \pm 0.90$ & -0.756 & 0.457 & $0.05 \pm 0.22$ & $-0.04 \pm 0.25$ & 1.739 & 0.193 \\
\hline $\mathrm{FEV}_{1} \mathrm{~L}$ & $1.63 \pm 0.66$ & $1.70 \pm 0.64$ & 1.441 & 0.162 & $1.64 \pm 0.67$ & $1.62 \pm 0.63$ & -1.223 & 0.233 & $0.07 \pm 0.29$ & $-0.04 \pm 0.15$ & 3.251 & 0.077 \\
\hline $\mathrm{FEV}_{1} \%$ predicted & $58.58 \pm 20.95$ & $59.92 \pm 19.24$ & 1.360 & 0.186 & $58.96 \pm 19.40$ & $58.73 \pm 20.21$ & -0.632 & 0.533 & $1.34 \pm 5.95$ & $-0.62 \pm 4.96$ & 2.063 & 0.157 \\
\hline FEV $_{1} /$ FVC $\%$ & $71.45 \pm 12.17$ & $72.20 \pm 13.94$ & 0.650 & 0.522 & $69.33 \pm 15.76$ & $70.21 \pm 11.66$ & 0.383 & 0.705 & $0.74 \pm 7.00$ & $0.97 \pm 12.90$ & 1.701 & 0.198 \\
\hline Inspiratory capacity L & $1.57 \pm 0.61$ & $1.71 \pm 0.65$ & -0.352 & 0.058 & $1.67 \pm 0.70$ & $1.68 \pm 0.60$ & -0.352 & 0.728 & $0.14 \pm 0.20$ & $-0.02 \pm 0.27$ & 5.399 & 0.024 \\
\hline PEF L.S $\mathrm{S}^{-1}$ & $4.11 \pm 1.48$ & $4.35 \pm 1.75$ & 1.991 & 0.728 & $4.29 \pm 1.74$ & $4.40 \pm 1.61$ & 0.181 & 0.858 & $0.24 \pm 0.71$ & $0.03 \pm 0.97$ & 1.165 & 0.285 \\
\hline $\begin{array}{l}\text { Sputum volume } \\
\mathrm{mL} \cdot 24 \mathrm{~h}^{-1}\end{array}$ & $45.19 \pm 37.62$ & $38.41 \pm 66.36$ & -0.858 & 0.399 & $43.52 \pm 45.47$ & $45.70 \pm 41.23$ & 0.420 & 0.678 & $-6.78 \pm 41.04$ & $2.19 \pm 27.01$ & 0.792 & 0.378 \\
\hline Sputum viscosity & $2.44 \pm 0.93$ & $3.11 \pm 0.85$ & -3.029 & 0.005 & $2.30 \pm 0.87$ & $2.74 \pm 0.81$ & -2.199 & 0.037 & $0.67 \pm 1.14$ & $0.44 \pm 1.05$ & 3.021 & 0.086 \\
\hline Purulent score & $6.04 \pm 0.98$ & $4.87 \pm 1.25$ & -5.691 & 0.001 & $5.46 \pm 1.38$ & $5.63 \pm 1.18$ & 0.774 & 0.446 & $-1.17 \pm 1.07$ & $0.17 \pm 1.12$ & 19.989 & 0.001 \\
\hline \multicolumn{13}{|l|}{ LCQ score } \\
\hline Physical & $4.40 \pm 1.16$ & $5.04 \pm 1.10$ & 3.119 & 0.004 & $4.43 \pm 1.14$ & $4.60 \pm 1.10$ & 0.660 & 0.515 & $0.65 \pm 1.08$ & $0.17 \pm 1.34$ & 2.736 & 0.104 \\
\hline Psychological & $3.48 \pm 1.23$ & $4.59 \pm 1.25$ & 5.640 & 0.001 & $3.86 \pm 1.10$ & $3.85 \pm 1.19$ & -0.031 & 0.975 & $1.11 \pm 1.03$ & $-0.01 \pm 1.28$ & 16.146 & 0.001 \\
\hline Social & $4.05 \pm 1.35$ & $4.81 \pm 1.38$ & 3.310 & 0.003 & $4.39 \pm 1.17$ & $4.13 \pm 1.37$ & -1.050 & 0.303 & $0.77 \pm 1.21$ & $-0.26 \pm 1.27$ & 9.855 & 0.003 \\
\hline Total score & $11.94 \pm 3.45$ & $14.45 \pm 3.57$ & 4.428 & 0.001 & $12.71 \pm 2.98$ & $12.60 \pm 3.49$ & -0.170 & 0.866 & $2.51 \pm 2.94$ & $-0.11 \pm 3.38$ & 11.386 & 0.001 \\
\hline 6MWD & $503.63 \pm 105.67$ & $529.48 \pm 115.67$ & 3.468 & 0.002 & $521.74 \pm 110.87$ & $497.44 \pm 141.21$ & -1.604 & 0.121 & $25.85 \pm 38.73$ & $-24.30 \pm 78.73$ & 9.618 & 0.003 \\
\hline \multicolumn{13}{|l|}{$\begin{array}{l}\text { Sputum cell } \\
\text { classification }\end{array}$} \\
\hline Neutrophils \% & $96.22 \pm 3.64$ & $95.99 \pm 5.45$ & -0.238 & 0.814 & $94.98 \pm 8.28$ & $97.02 \pm 2.19$ & 1.323 & 0.198 & $-0.23 \pm 4.94$ & $2.03 \pm 7.84$ & 1.458 & 0.233 \\
\hline Macrophages \% & $1.61 \pm 3.00$ & $1.19 \pm 1.72$ & -0.865 & 0.395 & $2.54 \pm 7.50$ & $1.03 \pm 1.37$ & -0.993 & 0.330 & $-0.42 \pm 2.46$ & $-1.52 \pm 7.78$ & 0.411 & 0.524 \\
\hline Eosinophils \% & $0.80 \pm 1.19$ & $1.53 \pm 3.90$ & 1.230 & 0.230 & $0.81 \pm 1.19$ & $0.86 \pm 1.16$ & 0.337 & 0.739 & $0.73 \pm 3.02$ & $0.05 \pm 0.73$ & 1.206 & 0.277 \\
\hline Lymphocytes \% & $1.37 \pm 2.26$ & $1.29 \pm 1.43$ & -0.180 & 0.858 & $1.63 \pm 1.62$ & $1.10 \pm 2.13$ & -1.177 & 0.250 & $-0.08 \pm 2.39$ & $-0.53 \pm 2.29$ & 0.494 & 0.485 \\
\hline $\begin{array}{l}\text { TNF- } \alpha \times 10^{3} \mathrm{pg} \cdot \mathrm{mL}^{-1} \\
(\mathrm{n}=17)\end{array}$ & $1.45 \pm 1.09$ & $1.26 \pm 1.37$ & 0.426 & 0.676 & $1.81 \pm 2.15$ & $1.43 \pm 0.91$ & 0.751 & 0.463 & $-0.19 \pm 1.81$ & $-0.39 \pm 2.11$ & 0.289 & 0.598 \\
\hline $\begin{array}{l}\mathrm{IL}-8 \times 10^{3} \mathrm{pg} \cdot \mathrm{mL}^{-1} \\
(\mathrm{n}=16)\end{array}$ & $273.25 \pm 158.15$ & $278.21 \pm 440.75$ & -0.042 & 0.967 & $444.02 \pm 515.37$ & $289.16 \pm 157.51$ & 1.426 & 0.174 & $4.95 \pm 473.71$ & $-154.86 \pm 434.31$ & 1.532 & 0.236 \\
\hline $\begin{array}{l}\mathrm{IL}-6 \times 10^{3} \mathrm{pg} \cdot \mathrm{mL}^{-1} \\
(\mathrm{n}=12)\end{array}$ & $0.87 \pm 0.90$ & $0.60 \pm 0.70$ & 1.059 & 0.312 & $0.57 \pm 0.88$ & $0.47 \pm 0.55$ & 0.394 & 0.701 & $-0.28 \pm 0.91$ & $-0.10 \pm 0.87$ & 0.306 & 0.592 \\
\hline
\end{tabular}

Data are presented as mean \pm SD, unless otherwise stated. UNW: $0.9 \%$ normal saline inhaled by ultrasonic nebuliser with warming; FVC: forced vital capacity; FEV ${ }_{1}$ : forced expiratory

volume in $1 \mathrm{~s}$; PEF: peak expiratory flow; LCQ: Leicester cough questionnaire; 6MWD: 6-min walk distance; TNF: tumour necrosis factor; IL: interleukin. 


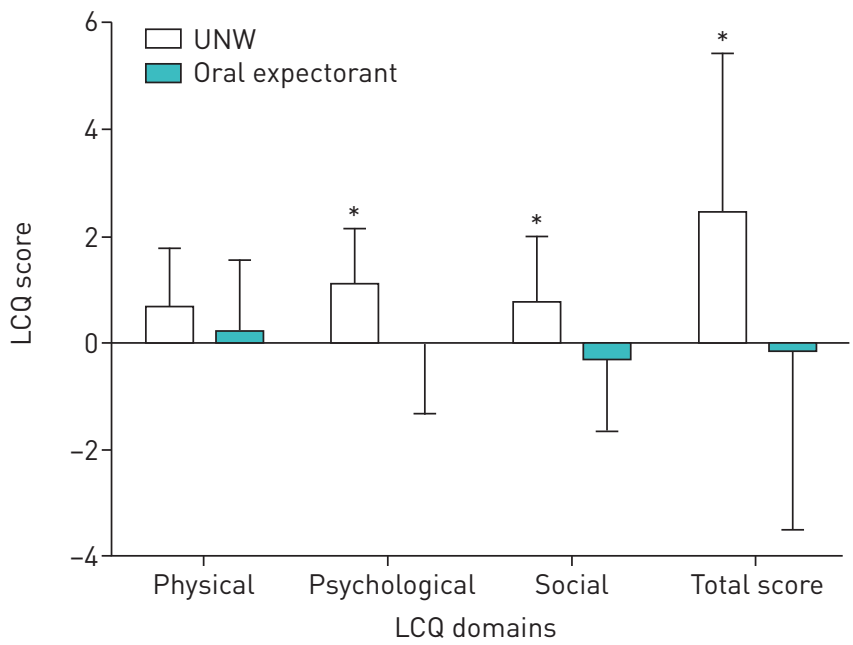

FIGURE 3 Change in the Leicester cough questionnaire (LCQ) individual domain and total scores with ultrasonic nebulisation with warming (UNW) and with oral expectorant treatment. *: $p<0.05$.

The absolute humidity of the gas is proportional to the temperature, because the temperature of the airway cannot exceed $37^{\circ} \mathrm{C}$, and most patients can only inhale the gas at a temperature of $32-35^{\circ} \mathrm{C}$. Therefore, the other way to increase the absolute humidity of the gas is to include atomised droplets. When the $32-35^{\circ} \mathrm{C}$ gas passes through the oropharynx and larynx, the temperature rises. The atomised droplets in the gas are heated and evaporate, ensuring that the relative humidity of the inhaled gas reaches $100 \%$ at $37^{\circ} \mathrm{C}$.

When inhaling atomised gas at a temperature of $<20^{\circ} \mathrm{C}$, the airway mucosa consumes a lot of heat to heat the atomised droplets, and the airway temperature decreases significantly. This low temperature not only stimulates airway mucosa to cause airway spasm and cough, but also reduces the ability of the mucosal cilia to oscillate [16]. Inhalation of atomised gas at room temperature is not ethical; therefore, the control group was given the oral expectorants most commonly used for NCFB, instead of inhalation of normal saline at room temperature.

Sputum volume and purulence score are common clinical indexes of treatment efficacy in NCFB [8]. Furthermore, the sputum volume in $24 \mathrm{~h}$ reflects the level of disease control and acute exacerbation. Bronchiectasis is a neutrophil-based inflammatory disease of the airways [17]. The sputum colour represents the degree of sputum purulence, and reflects the level of catalase released by neutrophils. The daily sputum volume and colour are relatively stable in patients with stable bronchiectasis, as the degree of lung injury and inflammation are relatively stable in the short term. The present results showed that there was no significant difference in the sputum volume in the $24 \mathrm{~h}$ after UNW aerosol therapy.

However, compared with oral expectorant therapy, UNW resulted in a significantly better improvement in both sputum colour and viscosity. When UNW is applied, the sputum in the airway absorbs moisture

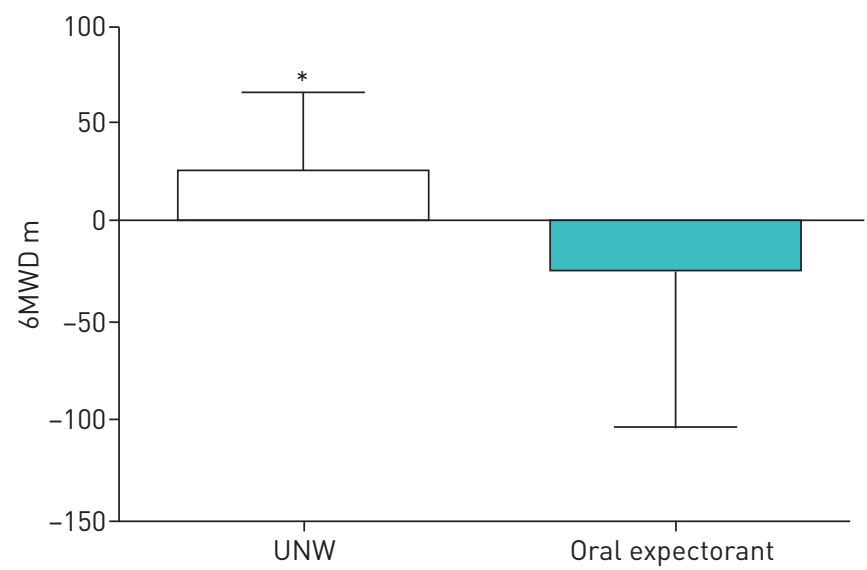

FIGURE 4 Change in the 6-min walk distance (6 MWD) with ultrasonic nebulisation with warming (UNW) and with oral expectorant treatment. *: $p<0.05$. 
from the inhaled gas, making the sputum lighter in colour and lower in viscosity. The volume of 24-h sputum after each treatment was not different, but if the moisture absorbed by the sputum is excluded, the patients treated with UNW should have had a lesser volume of sputum in $24 \mathrm{~h}$ than those treated with oral expectorant. After UNW aerosol therapy for 3 months, the airway sputum absorbed the moisture from the inhaled gas, was diluted, became mobile and was easily pushed upward by airway cilia and coughed out. The airway inflammation of bronchiectasis was improved because the sputum was fully cleared; as a result, airway sputum due to bronchiectasis was reduced. To investigate the effect of expectoration on airway inflammation, sputum cytology and airway inflammation markers (IL-6, IL-8 and tumour necrosis factor- $\alpha$ ) were measured before and after 3 months of treatment, but these markers did not change significantly after treatment. However, since bronchiectasis is a chronic suppurative inflammatory disease, 3 months of treatment may not be sufficient to improve the severity of inflammation $[8,18]$.

Improving the HRQoL is one of the main goals in the treatment of bronchiectasis. Repeated coughing might seriously decrease the HRQoL of patients. HRQoL for patients with chronic cough was assessed with the LCQ [9]. The LCQ can be used to assess the severity of bronchiectasis and to evaluate the clinical effect of treatment from physical, psychological and social domains [9]. Although the effect of cough on the psychological health in group A was greater than that in group B at their baselines, after 3 months of treatment, the total LCQ score and its three domain scores (physical, psychological and social) in the UNW treatment group improved, while those in the oral expectorant treatment group did not. This suggests that UNW could improve the HRQoL of patients with bronchiectasis. These findings are consistent with the results of a previous study of 40 patients with bronchiectasis treated by atomised gas inhalation [19]. The improvement of HRQoL in patients with bronchiectasis after UNW treatment may be due to easier clearance of sputum and relief of cough symptoms.

Acute exacerbation of repeated bronchiectasis leads to repeated aggravation of airway inflammation and leads to and thickening of the bronchial wall, destruction of lung parenchyma and damage of pulmonary ventilation function. Sputum in the airways may also cause obstructive ventilation dysfunction. After the sputum is cleared, the sputum-associated obstruction is alleviated or eliminated, and so the pulmonary ventilation function is improved. However, in cases with little sputum, there is no obvious sputum-associated airway obstruction; therefore, clearing the sputum does not improve the pulmonary ventilation function. This may be the reason for the inconsistency in the effect of inhaling humidified gas to clear airway sputum on improving pulmonary ventilation function in patients with bronchiectasis $[19,20]$.

The results showed that although there was no significant difference in the improvements of $\mathrm{FEV}_{1}$ and FVC between treatment with UNW versus oral expectorant, the improvement of inspiratory capacity after UNW was significantly greater than that after oral expectorant. This is consistent with the result that UNW significantly improved the colour of the sputum. Collectively, the results suggest that UNW was superior to oral expectorant, and improved the airway obstruction caused by sputum. Furthermore, inspiratory capacity is related to athletic endurance [21, 22]. Compared with baseline, 3-month UNW treatment significantly improved 6MWD and the effect was significantly better than that of oral expectorant treatment.

Insufficient drainage of sputum can aggravate airway obstruction and airway inflammation, which may be one of the causes of recurrent acute exacerbation of bronchiectasis. In this study, the frequency of acute exacerbation decreased significantly from $0.85 \pm 0.91$ exacerbations 3 months before UNW treatment to $0.41 \pm 0.69$ exacerbations 3 months after UNW treatment. Additionally, the frequency of acute exacerbation at 3 months was reduced compared with oral expectorant treatment. However, possibly due to the insufficient number of cases there is no statistically significant difference. This suggests that inadequate drainage of purulent sputum may lead to repeated exacerbation of bronchiectasis. Larger studies are needed to validate the results and explore the potential benefits of UNW.

In the present study, gastrointestinal reactions occurred in two patients during oral expectorant therapy; this may be related to the side-effects of the oral expectorant drug. One patient developed chest tightness during treatment, which disappeared after inhalation of short-acting bronchodilators and active coughing to remove sputum. This chest tightness may be due to hypoventilation caused by sputum expansion after inhalation of humidified gas. After that, during the UNW treatment period, patients were encouraged to cough actively to clear sputum every five breathing cycles, and chest tightness did not occur again.

The device of ultrasonic nebulisation with warming was developed specifically for patients with viscous purulent phlegm. It provides atomised gas with supersaturated humidity at temperatures of $32-35^{\circ} \mathrm{C}$ determined by previous research experience. As a unique and simple system, enabling patients with NCFB to promote sputum excretion, and being easy to use, without skilled training or supervision, the new device is home-based, and can potentially be incorporated as a self-administered technique, adding to 
current NCFB treatments. The limitations of this study include the small number of cases and only 3 months of observation. It requires more observational subjects, multicentre and $\geqslant 1$-year studies to further confirm the impact of UNW on inflammatory markers, lung function, quality of life, frequency of acute exacerbation and prognosis in patients with bronchiectasis

\section{Conclusions}

3 months of UNW treatment significantly improved sputum drainage, inspiratory capacity, HRQoL and exercise endurance, and decreased the acute exacerbation frequency in patients with NCFB, but did not significantly improve the levels of inflammatory markers.

Acknowledgements: We thank S. Birring (King's College Hospital, London, UK) for permitting us to use the Leicester cough questionnaire. We also thank all of the patients who participated in the study. We thank Kelly Zammit from Liwen Bianji, Edanz Group China, for editing the English text of a draft of this manuscript, and Guansheng Su (State Key Laboratory of Respiratory Disease, Guangzhou, China) for his polish and revision assistance during the preparation of this manuscript.

Conflict of interest: L. Zhong has nothing to disclose. Y. Xiong has nothing to disclose. Z. Zheng reports grants from National Science and Technology Major Project and a project of National Natural Science Foundation of China during the conduct of the study. In addition, Dr Zheng has a patent ZL201220412530.3 licensed. N. Liu has nothing to disclose. J. Hu has nothing to disclose. F. Yang has nothing to disclose. R. Chen has nothing to disclose.

Support statement: This study was funded by National Science and Technology Major Project (No. 2016YFC1304600) and National Natural Science Foundation of China (No. 81670041). Funding information for this article has been deposited with the Crossref Funder Registry.

\section{References}

Barker AF. Bronchiectasis. N Engl J Med 2002; 346: 1383-1393.

2 Expert Consensus Group for Diagnosis and Treatment of Adult Bronchiectasis. Expert consensus on diagnosis and treatment of adult bronchiectasis. Chin J Tuberc Respir Dis 2012; 35: 485-492.

3 Foster WM. Mucociliary transport and cough in humans. Pulm Pharmacol Ther 2002; 15: 277-282.

4 Balsamo R, Lanata L, Egan CG. Mucoactive drugs. Eur Respir Rev 2010; 19: 127-133.

5 Ji YR, Kim HS, Park JH. Instillation of normal saline before suctioning in patients with pneumonia. Yonsei Med J 2002; 43: 607-612.

6 Davies MW, Dunster KR, Cartwright DW. Inspired gas temperature in ventilated neonates. Pediatr Pulmonol 2004; 38: 50-54.

7 Luo Q, Zheng $\mathrm{Z}$, Cen $\mathrm{H}$, et al. A modified nebulization modality versus classical ultrasonic nebulization and oxygen-driven nebulization in facilitating airway clearance in patients with acute exacerbation of chronic obstructive pulmonary disease: a randomized controlled trial. J Thorac Dis 2015; 7: 1130-1114.

8 Pasteur MC, Bilton D, Hill AT, et al. British Thoracic Society guideline for non-CF bronchiectasis. Thorax 2010 65: i1-i58.

9 Murray MP, Turnbull K, MacQuarrie S, et al. Validation of the Leicester Cough Questionnaire in non-cystic fibrosis bronchiectasis. Eur Respir J 2009; 34: 125-131.

10 Tsang KW, Tan KC, Ho PL, et al. Inhaled fluticasone in bronchiectasis: a 12 month study. Thorax 2005; 60: 239-243.

11 ATS Committee on Proficiency Standards for Clinical Pulmonary Function Laboratories. ATS statement guidelines for the six-minute walk test. Am J Respir Crit Care Med 2002; 166: 111-117.

12 Cole PJ. Inflammation: a two-edged sword - the model of bronchiectasis. Eur J Respir Dis Suppl 1986; 147: 6-15.

13 Shen Y, Huang S, Kang J, et al. Management of airway mucus hypersecretion in chronic airway inflammatory disease: Chinese expert consensus. Int J Chron Obstruct Pulmon Dis 2018; 30: 399-407.

14 Williams R, Rankin N, Smith T, et al. Relationship between the humidity and temperature of inspired gas and the function of the airway mucosa. Crit Care Med 1996; 24: 1920-1929.

15 Hasani A, Chapman TH, McCool D, et al. Domiciliary humidification improves lung mucociliary clearance in patients with bronchiectasis. Chron Respir Dis 2008; 5: 81-86.

16 Bathoorn E, Liesker J, Postma D, et al. Safety of sputum induction during exacerbations of COPD. Chest 2007; 131: 432-438.

17 Stockley RA, Bayley D, Hill SL, et al. Assessment of airway neutrophils by sputum colour: correlation with airway inflammation. Thorax 2001; 56: 366-372.

18 Rogers GB, van der Gast CJ, Cuthbertson L, et al. Clinical measures of disease in adult non-CF bronchiectasis correlate with airway mirobiota composition. Thorax 2013; 68: 731-737.

19 Nicolson CH, Stirling RG, Borg BM, et al. The long term effect of inhaled hypertonic saline 6\% in non-cystic fibrosis bronchiectasis. Respir Med 2012; 106: 661-667.

20 Elkins MR, Robinson M, Rose BR, et al. A controlled trial of long-term inhaled hypertonic saline in patients with cystic fibrosis. N Engl J Med 2006; 354: 229-240.

21 Koulouris NG, Dimopoulou IP, Valta P, et al. Detection of expiratory flow limitation during exercise in COPD patients. J Appl Physiol 1997; 82: 723-731.

22 Cui L, Ji X, Xie M, et al. Role of inspiratory capacity on dyspnea evaluation in COPD with or without emphysematous lesions: a pilot study. Int J Chron Obstruct Pulmon Dis 2017; 12: 2823-2830. 